\section{(2) OPEN ACCESS}

\title{
Perceived efficacy of HIV treatment-as-prevention among university students in Johannesburg, South Africa
}

\author{
Jacob Bor (1) , ${ }^{1,2,3}$ Nozipho Musakwa, ${ }^{3}$ Dorina Onoya, ${ }^{3}$ Denise Evans ${ }^{3}$
}

\begin{abstract}
- Additional supplemental material is published online only. To view, please visit the journal online (http://dx.doi. org/10.1136/sextrans-2021055031).
\end{abstract}

'Department of Global Health, Boston University School of Public Health, Boston, Massachusetts, USA ${ }^{2}$ Department of Epidemiology, Boston University School of Public Health, Boston, Massachusetts, USA ${ }^{3}$ Health Economics and Epidemiology Research Office, Wits Health Consortium, Department of Internal Medicine, School of Clinical Medicine, Faculty of Health Sciences, University of the Witwatersrand, Johannesburg, Gauteng Province, South Africa

\section{Correspondence to}

Dr. Jacob Bor, Department of Global Health, Boston University School of Public Health, Boston, MA 02118, USA; jbor@bu.edu

Received 23 February 2021 Accepted 9 June 2021

Published Online First 11 September 2021

\section{Check for updates}

(C) Author(s) (or their employer(s)) 2021. Re-use permitted under CC BY-NC. No commercial re-use. See rights and permissions. Published by BMJ.

\section{To cite: Bor J,}

Musakwa N, Onoya D,

et al. Sex Transm Infect

2021:97:596-600.

\begin{abstract}
Objective Antiretroviral therapy (ART) nearly eliminates HIV transmission. Yet information on treatment as prevention (TasP) has been slow to diffuse in subSaharan Africa. We assessed TasP knowledge among university students in South Africa.

Methods We conducted a cross-sectional survey of first-year university students at a large public university in Johannesburg, South Africa, all of whom would have recently completed secondary school HIV curricula. Respondents were asked to consider the likelihood of HIV transmission in a serodiscordant couple having condomless sex with and without virally suppressive ART. Beliefs were elicited using a 0-20 visual scale. Perceived TasP efficacy was computed as the relative reduction in risk associated with virally suppressive ART. We compared beliefs with estimates from the scientific literature and assessed associations with demographics, HIV testing history and qualitative measures of HIV knowledge and risk perception.
\end{abstract}

Results The analysis included 365 university students ages $18-25$ years (48\% female, $56 \%$ from Gauteng Province). On average, perceived annual risk of HIV transmission with virally suppressive ART was $73 \%$; the objective risk is $<1 \%$. On average, respondents perceived that virally suppressive ART reduced annual transmission risk by $17 \%$; the objective reduction in risk is $>96 \%$. We observed no differences in perceived TasP efficacy by participant characteristics and testing history. Perceived TasP efficacy was correlated with the (correct) belief that HIV risk increases with sexual frequency.

Conclusions University students in South Africa underestimated the prevention benefits of HIV treatment. Low knowledge of TasP could limit demand for HIV testing and treatment among young adults.

\section{INTRODUCTION}

The HIV Prevention Trials Network (HPTN)-052 trial $^{12}$ and multiple large cohort studies ${ }^{34}$ showed that antiretroviral therapy (ART) eliminates HIV transmission if the infected partner is virally suppressed. Treatment as prevention (TasP) has motivated countries to expand ART coverage in order to reduce HIV incidence. ${ }^{5}$ However, there remain significant gaps in HIV testing and treatment, particularly among young adults. ${ }^{6-8}$

Despite scientific consensus on the efficacy of TasP, little is known about the diffusion of TasP information to young adults in countries where
HIV is endemic. Studies have found increases in TasP knowledge in the 2010s among male sexual minority populations in North America, Europe and Australia. ${ }^{9-12}$ However, data from sub-Saharan Africa are scarce. ${ }^{13}$ Evidence from South Africa, Zambia and Uganda suggest low familiarity with TasP in rural areas. ${ }^{14-16}$ However, information may have diffused faster in urban areas, among young adults, and among those with access to secondaryschool HIV education and university health services.

We set out to measure beliefs about TasP among first-year university students in Johannesburg, South Africa. All South African university students would have received HIV education throughout their primary and secondary schooling, as South Africa has provided HIV education under the Integrated School Health Programme since 2000. ${ }^{17}$ We elicited participants' beliefs about the probability of HIV transmission in a serodiscordant couple, varying whether the HIV-infected partner was on ART and virally suppressed. We computed TasP efficacy as the perceived reduction transmission risk with ART. We assessed the association of perceived TasP efficacy with respondent characteristics and beliefs.

\section{METHODS}

\section{Procedures}

We surveyed first-year students, aged 18-25 years, between August and October 2017 at a large, public university in Johannesburg, South Africa. ${ }^{17}$ Students in common areas of university campus (eg, library, canteen and lunch area) were invited to participate. We excluded students who had completed secondary school more than 3 years ago, those who had been a university student for more than 1 year and those who were not comfortable communicating in English. After providing written informed consent, study participants completed a self-administered, paper-based questionnaire. This study was nested as part of a larger study, the HIV/ TB Knowledge, Risk Perceptions and Barriers to Accessing Care study. ${ }^{17}$

\section{Measures}

We assessed beliefs about the likelihood of HIV transmission in four scenarios using a Visual Analogue Scale (online supplemental table 1). ${ }^{12}$ Participants were asked: 'Consider a woman who does not have HIV. Imagine she has sex one time 
with a man who is HIV-infected and they do not use a condom. Choose a number from 0 to 20 to reflect how likely you think it is that she will become infected with HIV'. Participants were also asked to consider the likelihood after a year of weekly condomless sex (52 times). We then asked participants to consider the likelihood of HIV transmission (after 1 and 52 sex acts) in an alternate scenario 'with a man who is HIV-infected but who is taking ARVs every day and is virally suppressed'. We transformed all responses to a $0 \%-100 \%$ scale, multiplying by 5 . We defined perceived efficacy of TasP as the relative (percent reduction in transmission risk due to virally suppressive ART after 52 condomless sex acts.

The four transmission scenarios were chosen to reflect realistic situations that we could benchmark to the scientific literature. In a meta-analysis of studies from low-income countries, the peract HIV transmission probability from a man to a woman was estimated to be $0.3 \%{ }^{18}$ After 52 condomless sex acts, the probability of HIV acquisition is $14.5 \%$, computed as $1-(1-0.003)^{52}$. The HPTN-052 trial found a 96\% reduction in infection risk, ${ }^{19}$ implying a $0.01 \%$ risk after 1 sex act and a $0.6 \%$ risk of HIV acquisition after 52 condomless sex acts with a partner on ART and virally suppressed. The PARTNERS and Opposites Attract studies indicate the true transmission risk when virally suppressed is zero (or very close to zero). ${ }^{34}$

Data were additionally collected on respondents' age, gender, location of secondary school, receipt of financial aid, residence in student housing, source of health insurance, food insecurity, and an asset index. ${ }^{20}$ Participants were asked when they last had an HIV test. HIV knowledge was assessed using an index of 42 items, ${ }^{11}$ split at the median into high and low knowledge. HIV risk perception was assessed using an abridged version of the Napper Scale (online supplemental table 2), ${ }^{21}$ split at the median into low-risk and high-risk perception. Finally, we collected data on beliefs related to the effect of sexual frequency and ART on transmission (online supplemental table 3). Details on all measures are described elsewhere. ${ }^{17}$

\section{Data analysis}

We plotted the distributions of perceived likelihood of HIV transmission in the different scenarios and we computed the mean and 10th, 25th, 50th, 75th and 90th percentiles of these distributions. We assessed absolute differences in perceived transmission risk associated with frequency of sex acts (1 condomless sex act vs 52 condomless sex acts) and with virally suppressive ART (52 condomless sex acts vs 52 condomless sex acts on ART). We summarised perceived TasP efficacy in two ways. First, we computed the population-average perceived TasP efficacy: 100\%-100\% $\times$ (average perceived 1-year transmission risk with ART/average perceived 1-year transmission risk without ART). This quantity is most comparable to the HPTN-052 trial, which reported the ratio of infection rates in treated and control groups. Second, we computed individual-level perceived TasP efficacy as the percent reduction in perceived transmission risk with ART for each respondent. Some participants reported higher transmission likelihood with ART, compared with without ART; these responses were bottom-coded at $0 \%$ prevention efficacy of TasP. We plotted the distribution of individual-level perceived TasP efficacy as a histogram and assessed differences in perceived TasP efficacy by respondent characteristics and beliefs. Stata/SE V14.2 was used for all analyses.(online supplemental files 2, 3)
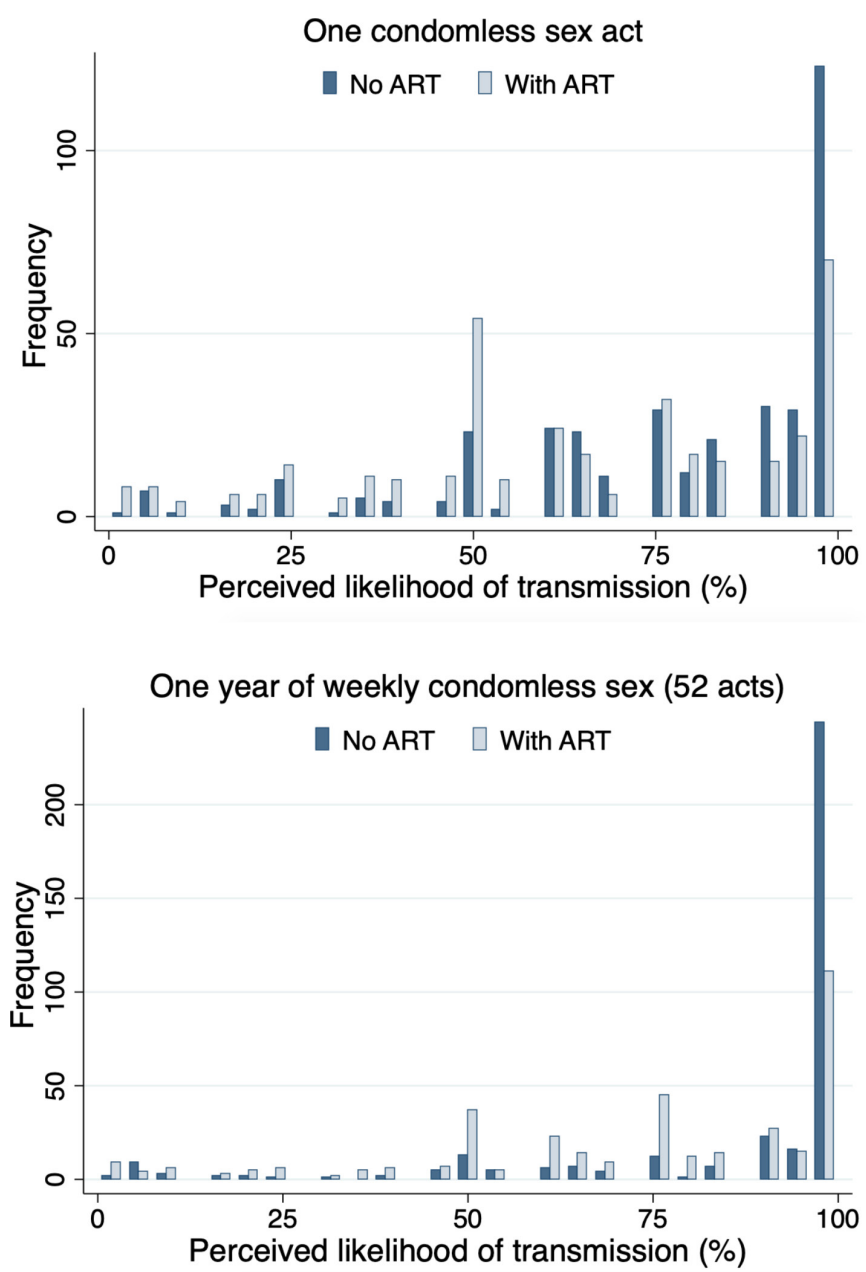

Figure 1 Perceived likelihood of HIV transmission in four scenarios. Note: $n=365$. The distribution of beliefs about the likelihood that a woman would become infected with HIV is shown if she had condomless sex with an HIV-infected male partner in four scenarios: (top) one sex act with versus without virally suppressive ART and (bottom) 52 sex acts with versus without virally suppressive ART. ART, antiretroviral therapy.

\section{RESULTS}

\section{Respondent characteristics}

A total of 365 students were included in the analysis. The sample was $48 \%$ female; $45 \%$ were ages $18-19$ years (53\% were $20-25$ years); and 44\% attended secondary school outside of Gauteng Province. Just over half of respondents reported receiving a loan or scholarship to fund their tuition, and about half lived on campus in student housing (online supplemental table 3). The sample was $96 \%$ black African.

\section{Transmission risk in 1 vs 52 sex acts}

Respondents believed HIV transmission to be much more likely than reported in the scientific literature. Figure 1 shows the distributions of participants' transmission beliefs in the four scenarios. On average, respondents perceived a 78.3\% likelihood of transmission after one condomless sex act in a serodiscordant couple, and a $87.9 \%$ likelihood of transmission after 52 sex acts, compared with objective risks of $0.3 \%$ and $14.5 \%$ (table 1). Many respondents perceived similar risks from 1 and 52 sex acts. These belief patterns are consistent with responses to survey questions: $95.9 \%$ of respondents affirmed that someone 
Table 1 Perceived versus objective transmission risk under four scenarios

\begin{tabular}{|c|c|c|c|c|c|c|c|}
\hline \multirow[b]{2}{*}{ Parameter } & \multirow[b]{2}{*}{ Objective } & \multirow{2}{*}{$\begin{array}{l}\text { Perceived } \\
\text { (mean) }\end{array}$} & \multicolumn{5}{|c|}{ Perceived (percentile) } \\
\hline & & & 10th & 25 th & 50 th & 75th & 90th \\
\hline \multicolumn{8}{|l|}{ Risk of HIV transmission (\%) } \\
\hline Scenario 1: one sex act & 0.30 & 78.3 & 45 & 65 & 85 & 100 & 100 \\
\hline Scenario 2: one sex act with ART & 0.01 & 65.1 & 25 & 50 & 65 & 95 & 100 \\
\hline Scenario 3: 52 sex acts & 14.5 & 87.9 & 50 & 90 & 100 & 100 & 100 \\
\hline Scenario 4: 52 sex acts with ART & 0.62 & 73.1 & 35 & 55 & 75 & 100 & 100 \\
\hline \multicolumn{8}{|l|}{ Efficacy of TasP } \\
\hline Population average & $96 \%$ & $16.8 \%$ & & & & & \\
\hline Individual-specific & & $20.3 \%$ & $0 \%$ & $0 \%$ & $10 \%$ & $33 \%$ & $50 \%$ \\
\hline
\end{tabular}

Note: $n=365$. Table displays mean perceived transmission risk across the four scenarios in the study sample. Transmission risks are probabilities expressed as percentages.

Efficacy of TasP is computed as the relative (percent) reduction in transmission risk associated with ART. Objective risks are computed based on a uniform $0.3 \%$ per-act transmission risk ${ }^{18}$ and a $96 \%$ relative reduction in per-act transmission with ART. $^{1}$

ART, antiretroviral therapy; TasP, treatment as prevention .

can get HIV by having sex one time. Yet only 55.3\% agreed that having sexual intercourse less frequently reduced your risk of getting HIV (online supplemental table 3).

\section{Transmission risk with virally suppressive ART}

Participants perceived lower risk of transmission when the HIV-infected partner was on virally suppressive ART (figure 1); however, perceived risk remained high. Over three-quarters $(77 \%)$ of respondents perceived a $50 \%$ or greater likelihood of contracting HIV after just 1 sex act with a person with HIV who was on ART and virally-suppressed. The average perceived risk of transmission was $65.1 \%$ in 1 sex act and $73.1 \%$ in 52 sex acts when the HIV-positive partner was on ART and virally suppressed. The objective risk in these scenarios is zero (or very close to zero). The population-average perceived efficacy of TasP was $16.8 \%$, compared with the $96 \%$ reported in the HPTN-052 trial (table 1).

Figure 2 shows the distribution of individuals' beliefs about TasP efficacy on a relative scale (for risk differences, see online

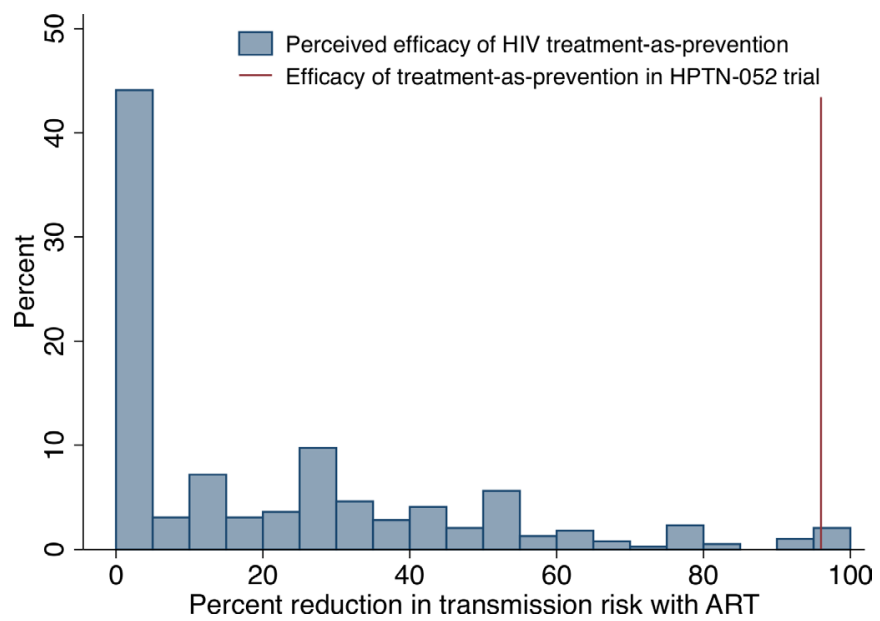

Figure 2 Perceived efficacy of HIV TasP. Note: Histogram displays the distribution of subject-specific beliefs about the prevention efficacy of ART, that is, the relative reduction in transmission risk with virally suppressive ART after 1 year of weekly condomless sex (52 times). Perceived TasP efficacy is defined as 100\%-100\% × (perceived transmission risk with ART/perceived transmission risk without ART). $0 \%$ reduction in risk includes respondents who reported higher transmission with ART than without ART. ART, antiretroviral therapy; TasP, treatment as prevention. supplemental figure 1). Two respondents reported zero risk of transmission without ART and thus TasP efficacy was not defined; they are excluded from the reported results. Of 363 respondents, $160(44.1 \%)$ did not report lower perceived transmission risk if the HIV-infected partner was on ART and virally suppressed. The median respondent perceived a $10 \%$ reduction in transmission risk with ART (table 1). Just 16.3\% (59/363) believed there was a $50 \%$ or greater reduction in transmission risk with virally suppressive ART, and just 2.8\% (10/363) of respondents believed that there was a $96 \%$ or greater reduction in risk.

Beliefs about TasP efficacy did not vary by demographic characteristics, socioeconomic status, HIV testing history nor HIV knowledge (online supplemental table 3). There was a modest and marginally significant increase in perceived efficacy among respondents who agreed with the statement: 'ART reduces transmission risk' $(p=0.075)$. Higher perceived TasP efficacy was associated with lower perceived HIV risk $(p=0.016)$. Higher perceived TasP efficacy was also associated with the belief that having less risk reduces infection risk $(\mathrm{p}<0.001)$, suggesting that an orientation towards a biological understanding of transmission may be important for people to assimilate information on TasP into their beliefs.

\section{DISCUSSION}

Nearly a decade after HPTN-052 showed that HIV treatment prevents transmission of the virus, young adults in South Africa remain largely unaware of these benefits. Although all South African university students would have received HIV education throughout their primary and secondary schooling, we found beliefs about HIV transmission that differed markedly from the scientific literature. We found that (1) respondents overestimated the probability of HIV transmission; (2) respondents underestimated the efficacy of HIV TasP; and (3) as a result, respondents vastly overestimated the likelihood of transmission when on ART and virally suppressed. The perceived annual risk of transmission with ART was 73\%. The objective risk is less than $1 \%$. As one respondent wrote, 'Taking ARVs doesn't matter. As long you are infected, it is very likely [for your partner] to get infected'.

Misperceptions about transmission risks are perhaps unsurprising. Historically, HIV education campaigns have exaggerated the virulence of $\mathrm{HIV}^{22}$ emphasising that you can get HIV from just one condomless sex act. Emphasis on this message may have contributed to the widespread belief ${ }^{23}$ that HIV transmission 
in one sex act is not just possible but likely. In many countries including South Africa, the prevention benefits of ART are not widely emphasised in public health messaging, school-based HIV curricula, nor HIV counselling. ${ }^{24}$ Although stable serodiscordant couples in HIV care are increasingly counselled on TasP, this represents a small fraction of young adults at risk. Consistent with the lack of emphasis on TasP in clinical settings, we found no differences in TasP knowledge by recent use of HIV testing nor by gender (despite gender disparities in care-seeking). Younger participants who were more recently in secondary school had marginally higher TasP knowledge than older participants, but the difference was not statistically significant.

Misperceptions may have significant impact on behaviour. Overestimating transmission may lead to fatalism ${ }^{25}$ and may contribute to greater HIV stigma and lower testing. ${ }^{26}$ Underestimating TasP efficacy could reduce the perceived benefits of treatment, particularly among people entering HIV care early in infection. ${ }^{27}$ Studies from the USA indicate that integrating TasP education into HIV counselling can improve adherence. ${ }^{28}$ In a cluster-randomised trial in Malawi, providing communities with information on TasP reduced stigma and increased HIV testing. ${ }^{29}$ A study in South Africa found that providing information on TasP increased HIV testing among men. ${ }^{30}$ Although there are reasons to be cautious in messaging on TasP, for example, potential for spread of other sexually-transmitted infections if people reduce condom use, providing information on the low risk of transmission on ART has potential to increase perceived benefits of ART, leading to greater treatment uptake, lower population viraemia, and fewer new HIV infections. ${ }^{31}$

Our analysis has some limitations. First, the study was a voluntary response sample. Although quite diverse in terms of demographic and socioeconomic characteristics, the sample was not designed to be representative of all students at the university, nor of all university students in South Africa. Second, it is possible that respondents may have interpreted the transmission probability questions in a qualitative way, reporting general feelings about the likelihood of an event rather than beliefs about the statistical probability of it occurring. Nevertheless, these data offer a more fine-grained picture of transmission beliefs than previously reported for this population.

A recent systematic review found that 31 studies measured TasP knowledge globally, from 2008 to $2020 .{ }^{13}$ However, just

\section{Key messages}

- HIV treatment prevents onward transmission of the virus; however, it is unknown whether information about HIV treatment-as-prevention has diffused to young adults in South Africa.

- We conducted a cross-sectional survey of first-year university students to determine beliefs about HIV transmission with and without virally suppressive HIV treatment.

- Respondents perceived a $73 \%$ annual transmission risk in a serodiscordant couple even if the HIV-infected partner was virally suppressed; the true risk is $<1 \%$.

- Respondents perceived that HIV treatment reduced transmission by $17 \%$ on average, much lower than the 96\% efficacy demonstrated in the HIV Prevention Trials Network-052 trial.

- Gaps in knowledge about HIV treatment-as-prevention may contribute to suboptimal care-seeking and persistent HIV stigma among young adults. two were quantitative studies of community-based samples in sub-Saharan Africa. In 2013, 65\% of survey participants in rural Malawi perceived that ART had no impact on transmission risk. ${ }^{29}$ In 2017, young adults in rural South Africa perceived a $75 \%$ annual risk of HIV transmission in a mixed status couple using TasP, similar to the findings in this study. ${ }^{32}$ Our study participants were urban university students who had all recently completed the secondary school curriculum in South Africa-a population one might expect to have high access to health information. To our knowledge, this the first study on TasP knowledge in an urban African setting.

In South Africa, young adults are at high risk of HIV infection and have among the lowest rates of engagement with HIV care. ${ }^{7}$ Addressing knowledge gaps regarding the prevention benefits of treatment through campaigns such as Undetectable $=$ Untransmittable $(\mathrm{U}=\mathrm{U})$ could encourage greater HIV testing and care-seeking in this population. Educational institutions offer valuable opportunities to provide accurate information to young adults about the benefits and limitations of TasP and to reduce stigma associated with HIV treatment.

\section{Handling editor Adam Huw Bourne}

Acknowledgements The authors thank the staff and students at the universities who supported and participated in the study. A special note of thanks to Alice Kono, Busi Sithole, Melda Musina, Portia Ngwenya, Vinolia Ntjikelene, Barbara Xhosa and Given Malete for their support and help with data collection, quality assurance and data management

Contributors JB and DE jointly conceptualised the study. DE designed and oversaw the study procedures. JB designed the survey instruments and analysis plan with input from DO. NM oversaw data collection and management. DE, JB, and NM wrote the first draft. All authors contributed to critically revising the paper, gave final approval of the version to be published and agreed to be accountable for all aspects of the work. JB is the study's guarantor.

Funding Funding for this study was provided by National Institutes of Health National Institute of Mental Health awards R34-MH122323 (JB) and K01MH105320 (JB) and Eunice Kennedy Shriver National Institute of Child Health and Human Development award R01-HD084233 (JB). The contents of the article are the responsibility of the authors and do not necessarily reflect the views of the US government. The funders had no role in the study design, collection, analysis and interpretation of the data, in manuscript preparation or the decision to publish.

Competing interests None declared

\section{Patient consent for publication Not required.}

Ethics approval The study was approved by the human research ethics committee (medical) of the University of the Witwatersrand (certificate number M161019).

Provenance and peer review Not commissioned; externally peer reviewed.

Data availability statement All data relevant to the study are included in the article or uploaded as supplementary information. Stata data and .do file are available as supplementary information for replication purposes.

Open access This is an open access article distributed in accordance with the Creative Commons Attribution Non Commercial (CC BY-NC 4.0) license, which permits others to distribute, remix, adapt, build upon this work non-commercially, and license their derivative works on different terms, provided the original work is properly cited, appropriate credit is given, any changes made indicated, and the use is non-commercial. See: http://creativecommons.org/licenses/by-nc/4.0/.

ORCID iD

Jacob Bor http://orcid.org/0000-0002-5112-8536

\section{REFERENCES}

1 Cohen MS, Chen YQ, McCauley M, et al. Prevention of HIV-1 infection with early antiretroviral therapy. N Engl J Med Overseas Ed 2011;365:493-505.

2 Cohen MS, Chen YQ, McCauley M, et al. Antiretroviral therapy for the prevention of HIV-1 transmission. N Engl J Med 2016;375:830-9.

3 Rodger AJ, Cambiano V, Bruun T, et al. Sexual activity without condoms and risk of HIV transmission in Serodifferent couples when the HIV-positive partner is using suppressive antiretroviral therapy. JAMA 2016;316:171 
4 Bavinton BR, Pinto AN, Phanuphak N, et al. Viral suppression and HIV transmission in serodiscordant male couples: an international, prospective, observational, cohort study. Lancet HIV 2018;5:e438-47.

5 WHO. Guideline on when to start antiretroviral therapy and on pre-exposure prophylaxis for HIV. Geneva: World Health Organization, 2015.

6 Maskew M, Bor J, MacLeod W. The youth treatment bulge in South Africa: increasing numbers, inferior outcomes among adolescents on ART. 21st International AIDS Conference, Durban, South Africa, 2016.

7 HSRC. Hiv impact assessment summary: the fifth South African national HIV prevalence, incidence, behaviour and communication survey (SABSSM V), 2018. Available: http://www.hsrc.ac.za/uploads/pageContent/9234/SABSSMV_Impact_ Assessment_Summary_ZA_ADS_cleared_PDFA4.pdf [Accessed 26 Mar 2019].

8 McBride K, Parent J, Mmanga K, et al. ART adherence among Malawian youth enrolled in teen clubs: a retrospective chart review. AIDS Behav 2019;23:2629-33.

9 Bavinton BR, Holt M, Grulich AE, et al. Willingness to act upon beliefs about "treatment as prevention" among Australian gay and bisexual men. PLoS One 2016;11:e0145847.

10 Card KG, Armstrong HL, Lachowsky NJ, et al. Belief in treatment as prevention and its relationship to HIV status and behavioral risk. J Acquir Immune Defic Syndr 2018;77:8-16.

11 Young I, Davis M, Flowers P, et al. Navigating HIV citizenship: identities, risks and biological citizenship in the treatment as prevention era. Health Risk Soc 2019;21:1-16.

12 Rendina HJ, Cienfuegos-Szalay J, Talan A, et al. Growing acceptability of undetectable = Untransmittable but widespread misunderstanding of transmission risk: findings from a very large sample of sexual minority men in the United States. J Acquir Immune Defic Syndr 2020;83:215-22.

13 Bor J, Fischer C, Modi M, et al. Changing Knowledge and Attitudes Towards HIV Treatment-as-Prevention and "Undetectable = Untransmittable" : A Systematic Review. AIDS Behav 2021;146.

14 Bond V, Hoddinott G, Viljoen L, et al. Good health and moral responsibility: key concepts underlying the interpretation of treatment as prevention in South Africa and Zambia before rolling out universal HIV testing and treatment. AIDS Patient Care STDS 2016;30:425-34.

15 Bor J, Barofsky J, Flanagan D. Beliefs about the benefits of HIV treatment in the era of "treat all": evidence from rural South Africa. Population Association of America Annual Meeting; April 27, 2018, Denver, C0, 2018.

16 Ndyabakira A, Chamie G, Emperador D, et al. Men's beliefs about the likelihood of Serodiscordance in couples with an HIV-positive partner: survey evidence from rural Uganda. AIDS Behav 2020;24:967-74.

17 Evans D, Musakwa N, Nattey C, et al. Knowledge, risk perception and access to healthcare services for HIV and tuberculosis among university students in Johannesburg, South Africa. S Afr J CH 2018;12:19-31.
18 Boily M-C, Baggaley RF, Wang L, et al. Heterosexual risk of HIV-1 infection per sexual act: systematic review and meta-analysis of observational studies. Lancet Infect Dis 2009;9:118-29.

19 Cohen MS, Chen YQ, McCauley M, et al. Prevention of HIV-1 infection with early antiretroviral therapy. N Engl J Med 2011;365:493-505.

20 Filmer D, Pritchett LH. Estimating wealth effects without expenditure data--or tears: an application to educational enrollments in states of India. Demography 2001;38:115-32.

21 Napper LE, Fisher DG, Reynolds GL. Development of the perceived risk of HIV scale. AIDS Behav 2012;16:1075-83.

22 Green EC, Witte K. Can fear arousal in public health campaigns contribute to the decline of HIV prevalence? J Health Commun 2006;11:245-59.

23 Delavande A, Kohler H-P. HIV / AIDS-related expectations and risky sexual behavior in Malawi 2011.

24 Onoya D, Mohjele I, Sineke T. Health provider perspectives on implementation of same day ART initiation 6 months after policy change in South Africa. International AIDS Conference (poster), Amsterdam NL, 2018

25 Sterck 0. HIV/AIDS and fatalism: should prevention campaigns disclose the transmission rate of HIV? J Afr Econ 2014;23:53-104.

26 Ikechebelu IJ, Udigwe GO, Ikechebelu N, et al. The knowledge, attitude and practice of voluntary counselling and testing (VCT) for HIVIAIDS among undergraduates in a polytechnic in Southeast, Nigeria. Niger J Med 2006;15:245-9.

27 Lasry A, Sansom SL, Wolitski RJ, et al. HIV sexual transmission risk among serodiscordant couples. AIDS 2014;28:1521-9.

28 Kalichman SC, Cherry C, Kalichman MO, et al. Mobile health intervention to reduce HIV transmission: a randomized trial of behaviorally enhanced HIV treatment as prevention (B-TasP). J Acquir Immune Defic Syndr 2018;78:34-42.

29 Derksen L, Van Oosterhout J. Love in the time of HIV: testing as a signal of risk, 2017. Available: https://6b68d1ac-a-62cb3a1a-s-sites.googlegroups.com/site/lauraderksen/ files/DerksenJMP.pdf?attachauth=ANoY7cqtsc7DCF9z8W4SVkXduHG_Ge7WJQI4 ktD6B3WNWbGBjoe00jSR23032M7YSSracHx PYyq4TKbkC6YY18Npx2S5w47HJva VkhfIFZU1wbZrLYZCDJmM4hsySamngPxsGWJ5g5cB0EnMV28K3K [Accessed 17 Dec 2017].

30 Smith P, Buttenheim A, Schmucker L. Undetectable=untransmittable $(U=U)$ messaging increases uptake of HIV testing among men: results from a pilot cluster randomized trial. medRxiv2020.

31 Calabrese SK, Mayer KH. Providers should discuss $\mathrm{U}=\mathrm{U}$ with all patients living with HIV. Lancet HIV 2019;6:e211-3.

32 Bor J, Barofsky J, Flanagan D. Beliefs about the benefits of HIV treatment in the era of treat all. Population Association of America Annual Meeting, Denver, 2018. 